\title{
Research on Location Problem of Electric Vehicle Charging Stations
}

\author{
Zhenping Li, Yulei Zhang* \\ School of Information, Beijing Wuzi University, Beijing, China \\ Email address: \\ Lizhenping66@163.com (Zhenping Li), zhangyuleilaba@163.com (Yulei Zhang) \\ ${ }^{*}$ Corresponding author
}

\section{To cite this article:}

Zhenping Li, Yulei Zhang. Research on Location Problem of Electric Vehicle Charging Stations. Science Journal of Applied Mathematics and Statistics. Vol. 4, No. 3, 2016, pp. 108-114. doi: 10.11648/j.sjams.20160403.14

Received: May 14, 2016; Accepted: May 25, 2016; Published: June 7, 2016

\begin{abstract}
This paper studies the location problem of electric vehicle charging station in the case that electric vehicles are used as commuter tools. Firstly, according to the length of the commuter road, the number of electric vehicles which would be used as commuter tools on the road, candidate charging stations and the maximum mileage of electric vehicles, a weighted network including two types of edges is constructed. Secondly, the location problem of electric vehicle charging station is transformed into a maximum covering problem of the weighted network. Then an integer nonlinear programming model for the location problem of electric vehicle charging station is formulated, the objective function of the mathematical model is to maximize covering the electric vehicles. A heuristic algorithm is designed to solve the model. Finally, we do simulation using a numerical example. The results show that the mathematical model and algorithm are effective in solving the location problem of electric vehicle charging station.
\end{abstract}

Keywords: Electric Vehicle Charging Station, Location, Charging Piles, Weighted Network, Mathematical Model, Heuristic Algorithm

\section{Introduction}

With the increasing tension of oil resources and increasingly serious air pollution problems, people begin to pay attention to new energy vehicles, electric vehicles have become the mainstream of the automotive industry.

In recent years, many cities accelerate to promote the use of EVs (electric vehicles) in various ways. In Beijing, for example, according to "The action of EV application plan in Beijing (In2014-2017)", Beijing will orderly promote units and individuals to buy EVs and vigorously promote the use of EVs in rental industry and public aspect of the city. The EVs are new energy vehicles, although they can't cause environmental pollution, EVs have short mileage and long charging time, so the rapid development of EVs depends on whether the charging station's planning and layout is reasonable. EV charging station location problem has important theoretical and practical significance.

Many scholars have studied EV charging station location problem from different views. Tang [1] analyzes several factors that influence charging station planning for EVs and then an income optimization model of charging station planning for EVs is developed. And the global searching ability of the particle swarm optimization algorithm is used to solve the model. Jia [2] developed the location model of EV charging station and the solution to this model is to use the simulated annealing algorithms with taking the maximization of charging station utility as the objective function and the traffic flow as the constraint condition. Chen [3] proposed an optimal model, which aims to minimize the investment and operation costs of the distribution network and EV charging stations. And the ITSE-PGA was used to solve the model.

Most of the above studies of EV charging station location problem minimize the operational cost of the charging station and the construction cost or maximized the profit obtained from the charging stations. Researchers paid little attention on convenience services for consumers and coverage of EV charging stations. Based on the background that the government vigorously promotes the use of EVs and constructs the charging stations, we should not only consider the benefits, but also consider how to maximize the needs of 
EVs users, how to improve the quality of service and how to make utility maximization, so as to accelerate the promotion of the use of EVs. There are not many research literatures about locating charging station from the view of the convenience for EV users. Huang [4] developed an integer programming model to minimize the construction cost while satisfying demand of electric power from refueling stations. Sun [5] developed a new dynamic model, named as the Spatio-temporal location model, with dual purpose of achieving minimum waiting time and maximum service accessibility for a given number of EV charging stations. Ge [6] proposed a planning method of EV charging station considering the users' convenience in order to solve the problem that the main function of $\mathrm{EV}$ charging stations is to provide users with a convenient charging service. Chu [7] formulated the location-allocation model of gradual covering electric vehicle charging stations based on the gradual covering and time satisfaction function. Chen [8] proposed a multi-objective model which provides extra options for locating and sizing of EV charging stations. Cavadas [9] proposed mathematical model for locating EV charging stations under the successive activities of the travelers. He [10] explored how to optimally locate public charging stations for EVs on a road network, considering drivers' spontaneous adjustments and interactions of travel and recharging decisions. Hosseini [11] proposed a recharging station location model with queue which considers capacity, recharging time, and waiting time. The presented model is a set covering and can easily become a maximum cover model with or without predefined activities. Johannes [12] proposed a decision support system for placing charging stations in order to satisfy the charging demand of electric taxi vehicles.

Although these studies are considered convenience of EVs charging stations to cover the needs of users as much as possible, most of these studies are based on the fast charge mode and little studies for slow charging mode. In slow charge mode, a complete electric vehicle charging process takes at least 6-8 hours. Thus, the general electric vehicle users are not willing to stop for slow charging their electric vehicles in the travel way, and the fast charging mode may have more serious damage for batteries of EVs, so many EV users want to have the convenience of charging station and enough time for slow charging of their electric vehicles.

Because the slow charging mode needs a long time and the EV mileage is short, most cities buy EVs as daily commuter tools. When the EVs are regarded as a commuter tools, the main running route is the line between residential areas and workplaces of EV users. Usually, EVs park at night in residential areas' parking lots and park during the day in workplaces' parking lots. And the charging piles are considered in charging station. So there is sufficient time for the EVs slow charging day or night. To encourage people purchase and use EVs, the government will invest some money each year to establish a certain number of charging stations. The problem is where should the charging stations be established and how many charging piles should be established in each charging station in order to service for as much as possible EV users. The EV charging station location problem will be transformed into a maximum covering location problem of weighted network, then the mathematical model is built and a heuristic algorithm is designed to solve the model.

\section{Mathematical Model of Charging Station Location Problem}

\subsection{Problem Description}

There are $m$ residential areas which correspond to $m$ parking lots and $n$ workplaces which correspond to $n$ parking lots in a city. Let $A_{1}, A_{2}, \ldots, A_{m}$ represent $m$ residential areas and $B_{1}, B_{2}, \ldots, B_{n}$ represent $n$ workplaces respectively. The government intends to invest some money in establishing slow charging stations in the city. Multiple charging piles can be established in every charging station and establishing each charging pile needs the same construction cost. The question is which candidate charging station should be selected and how many charging piles should be established to meet as much as possible EV users.

In order to establish the mathematical model of EV charging station location problem, we use points representing for the residential areas corresponding to the parking lot and workplaces corresponding to the parking lot. If the distance from residential area $A_{i}$ to workplace $B_{j}$ is no more than the half of EV's maximum mileage (i.e., $d_{i j} \leq \frac{D}{2}$ ), we connect a solid edge between $A_{i}$ and $B_{j}$, denote the edge weight by $r_{i j}$; If the distance from residential area $A_{i}$ to workplace $B_{j}$ is greater than the half of EV's maximum mileage and no more than the maximum mileage (i.e., $\left.\frac{D}{2}<d_{i j} \leq D\right)$, we connect a dotted edge between $A_{i}$ and $B_{j}$, and denote the edge weight by $r_{i j}$; If the distance from $A_{i}$ to $B_{j}$ is greater than maximum mileage (i.e., $d_{i j}>D$ ), we don't connect the edge. So the weighted network which describes the usage of EVs is constructed (see Fig. 1). In the weighted network, circle nodes and block nodes respectively represent the parking lots in residential areas and workplaces, the edges between two nodes (solid lines or dotted lines) represent that the EVs can be used as commuting tools between the two corresponding nodes, the edge weight represents the number of EVs used by people in the edge. Because the distance between two points corresponding to solid line is not more than the half of EV's maximum mileage, so the commuting EVs in this route need only charged either in residential area or workplace, which can meet the needs of round-trip commuting; the distance between two points corresponding to dotted line is greater than the half of EV's maximum mileage and not more than the maximum mileage, so the EVs after charging can only go one way in this edge. If the users in this route use EVs as commuter tools, the charging 
stations must be established both in residential area and workplace; If the distance between residential area and workplace is greater than maximum mileage, the EVs can not be used as commuter tools between the two nodes. The problem is where should the charging stations be established and how many charging piles should be established in each charging station in order to service for as much as possible EV users.

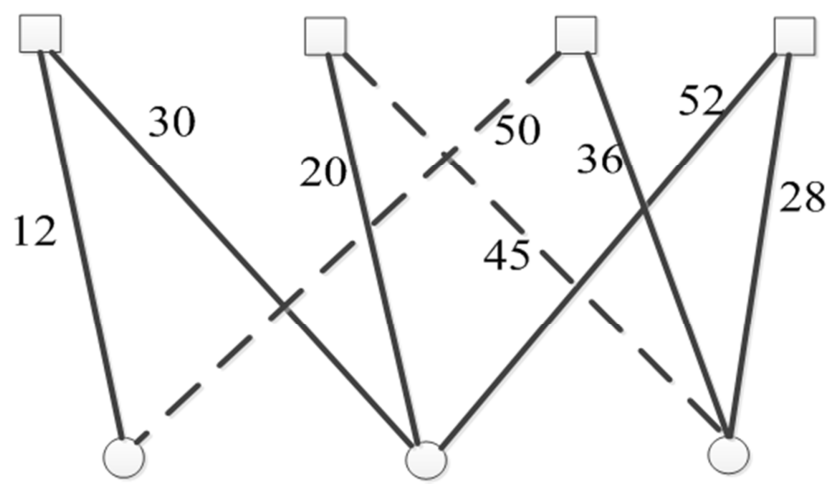

Fig. 1. Weighted internet graph about the use of EVS.

\subsection{Symbols and Definitions}

The symbols and variables are defined as follows: $m:$ Number of residential areas.

$n$ : Number of workplaces.

$d_{i j}$ : Distance between residential area $i$ and workplace $j$.

$D$ :Maximum mileage after the EV charging.

$C$ : Total cost (or budget) of building all charging stations.

$r_{i j}$ : The usage amounts of EVs between residential area $i$ and workplace $j$, where $j=1,2, \ldots, n, i=1,2, \ldots, m$.

$p_{i}$ : Maximum number of EVs which can be charged by one charging pile charging per day in charging station $i$.

$q_{j}$ : Maximum number of EVs which can be charged by one charging pile per day in charging station $j$.

$g_{i}$ : Cost of establishing a charging pile in residential area $i$.

$h_{j}$ : Cost of establishing a charging pile in workplace $j$.

$M$ : A large positive number.

$x_{i}$ : the number of charging piles established in charging station $i$.

$y_{j}$ : the number of charging piles established in charging station $j$.

$s_{i j}$ : Binary variable that takes 1 if $\mathrm{EV}$ user commuting between $i$ and $j$ can use charging stations, and 0 otherwise.

$a_{i}$ : Binary variable that takes 1 if charging station $i$ is built, and 0 otherwise.

$b_{j}$ : Binary variable that takes 1 if charging station $j$ is built, and 0 otherwise.

$w_{i j}$ : the weight of edge connecting node $i$ and node $j$.

$$
w_{i j}= \begin{cases}1, & d_{i j} \leq \frac{D}{2} \\ 2, & \frac{D}{2}<d_{i j} \leq D \\ 0, & d_{i j}>D\end{cases}
$$

\subsection{Mathematical Model}

Before the establishment of the mathematical model, two assumptions are taken into account. First, assume that the EV charging stations can only be established in parking lots. Second, each charging pile can service for limited EVs per day, the EVs exceeding the upper limit will not be charged properly.

If only one of the two parking lots connected by solid line establish a charging station, all EVs in the edge will be charged in the charging station, if both parking lots connected by solid line establish charging stations, each EV commuted in the edge chooses either charging station to charge by $50 \%$ probability. When both parking lots connected by dotted line establish charging stations, the EVs commuted in the edge need charged by both charging stations. When only one of the two parking lots connected by dotted line establishes a charging station, EVs in the edge will not be used as commuter tools and can't use the charging station.

The charging station location problem can be formulated into an integer programming model as follows:

$$
\begin{aligned}
& \max f=\sum_{i=1}^{m} \sum_{j=1}^{n} r_{i j} s_{i j} \\
& s . t\left\{\begin{array}{l}
w_{i j} s_{i j} \leq a_{i}+b_{j}, i=1,2, \ldots, m ; j=1,2, \ldots, n \\
\sum_{j\left(w_{i}=1\right)} r_{i j} a_{i}\left(1-b_{j} / 2\right)+\sum_{j\left(w_{i j}=2\right)} r_{i j} a_{i} b_{j} \leq p_{i} x_{i}+M\left(1-a_{i}\right), i=1,2, \ldots, m(3) \\
\sum_{j\left(w_{i}=1\right)} r_{i j} b_{j}\left(1-a_{i} / 2\right)+\sum_{j\left(w_{i j}=2\right)} r_{i j} b_{j} a_{i} \leq p_{i} x_{i}+M\left(1-a_{i}\right), j \\
\sum_{i=1}^{m} g_{i} x_{i}+\sum_{j=1}^{n} h_{j} y_{j} \leq C \\
x_{i} \leq M a_{i}, i=1,2, \ldots, m \\
y_{j} \leq M b_{j}, j=1,2, \ldots, n \\
x_{i} \geq 0, \text { int eger }, i=1,2, \ldots, m \\
y_{j} \geq 0, \text { int eger }, j=1,2, \ldots, n \\
s_{i j}=0,1, i=1,2, \ldots, m ; j=1,2, \ldots, n
\end{array}\right.
\end{aligned}
$$

The objective function (1) maximizes the EV users (or EVs) covered by charging station. Constraint (2) represents the condition of a route be covered by charging stations. If the route corresponds to a solid edge, at least one node of two ends establishes a charging station, if the route corresponds to a dotted edge, both nodes at two ends must establish charging stations. Constraints (3) and (4) represent the charging station's capacity restriction, i.e., the number of EVs served by a charging station doesn't exceed the maximum service capacity. Constraint (5) represents the sum of cost used to establish charging piles cannot exceed total invest. Constraints (6) and (7) represent that when some charging piles is built in a candidate site, the charging station is selected. Constraints (8) 
and (9) represent the value of decision variables.

\section{Heuristic Algorithm}

Because the mathematical model of EV charging station location problem is an integer nonlinear programming, it needs a long time to find the accurate solution, so we design a heuristic algorithm as follows:

Step 1: Construct a weighted network, the nodes of the network are the candidate EV charging stations. And the weight of each edge is the amounts of EVs used as commuter tools between its two adjacent nodes.

Step 2: Calculate total amounts of EVs commuting along the edge that can be effectively covered by each node. An edges is effectively covered by node $i$ means that it is a new covered edge after a charging station is established in node $i$, which includes two cases: (a) the solid edge adjacent to node $i$, (b) the dotted edge adjacent to node $i$ whose another node has established a charging station. Let $R_{i}$ be the total amounts of EVs which can be effectively covered by node $i$, and $u_{i}$ be the minimum number of charging piles established in node $i$ to cover $R_{i}$ EVs. Similarly, $R_{j}$ and $v_{j}$ are defined in node $j$. So the average costs of charging station $i$ covering one EV are $c u_{i}=\frac{u_{i} g_{i}}{R_{i}}$ and $c v_{j}=\frac{v_{j} h_{j}}{R_{j}}$ respectively.

Step 3: Sort the average costs $c u_{i}$ and $c v_{j}$ by increase order. Select the node with minimum average cost $c u_{i}$ or $c v_{j}$. Suppose node $k$ is the selected node, and $u_{k}$ (or $v_{k}$ ) are the corresponding charging piles in charging station $k$.

Step 4: Judge whether the total costs after establishing $u_{k}$ (or $v_{k}$ ) charging piles in charging station $k$ exceed the total costs $C$. If it doesn't exceed $C$, then establish $u_{k}$ (or $v_{k}$ ) charging piles in charging station $k$, and delete the solid edges and dotted edges that are effectively covered by new charging station $\mathrm{k}$. Go to step 2. Otherwise, determine the maximum number of charging piles which can be established in charging station $\mathrm{k}$ according to the total costs $C$, and establish the corresponding charging piles in charging station $\mathrm{k}$, go to step 5 .

Step 5: Output the number of charging piles established in every charging station. And calculate the total amount of EVs covered by all established charging stations.

\section{Analysis of an Example}

There are 6 residential areas and 4 workplaces in a city, see Figure 2. The circle nodes and block nodes respectively represent the residential areas (or parking lot in residential area) and workplaces (or parking lot in workplace).
(1)

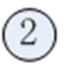

(3)
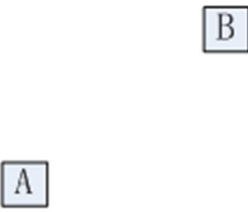

Fig. 2. A city's residential areas and workplaces distribution.

The number of EVs that might be used as commuter tools and commuting distance (unit:km) are as shown in Table 1.

Table 1. The number of EVs that might be used as commuter tools and commuting distance (unit: $\mathrm{km}$ ).

\begin{tabular}{lllll}
\hline Node & A & B & C & D \\
\hline 1 & $1(20)$ & $4(30)$ & $10(20)$ & $14(30)$ \\
2 & $8(10)$ & $7(20)$ & $4(30)$ & $20(20)$ \\
3 & $15(10)$ & $13(50)$ & $0(80)$ & $14(20)$ \\
4 & $14(15)$ & $0(75)$ & $12(20)$ & $0(80)$ \\
5 & $4(40)$ & $20(45)$ & $18(30)$ & $6(10)$ \\
6 & $25(20)$ & $9(10)$ & $5(5)$ & $10(20)$ \\
\hline
\end{tabular}

In order to encourage people purchase and use of EVs, the government is preparing to invest $\$ 1000$ to build charging stations in the city. Assume that $D=70, \frac{D}{2}=35$, $C=1000, \quad p=(10,10,10,10,10,10), \quad q=(20,20,20,20)$, $g=(60,70,80,100,60,90) \quad, \quad h=(80,100,70,90)$, $M=10000000, W$ is calculated by the commuting distance

$$
W=\left(\begin{array}{llll}
1 & 1 & 1 & 1 \\
1 & 1 & 1 & 1 \\
1 & 2 & 0 & 1 \\
1 & 0 & 1 & 0 \\
1 & 2 & 1 & 1 \\
1 & 1 & 1 & 1
\end{array}\right) .
$$

According to the number of EVs that might be used for commuting and commuting distance, the weighted network is constructed. The weights of edges represent the number of commuting EVs. If there are no people using EVs as commuter tools between two nodes, there is no edge. 


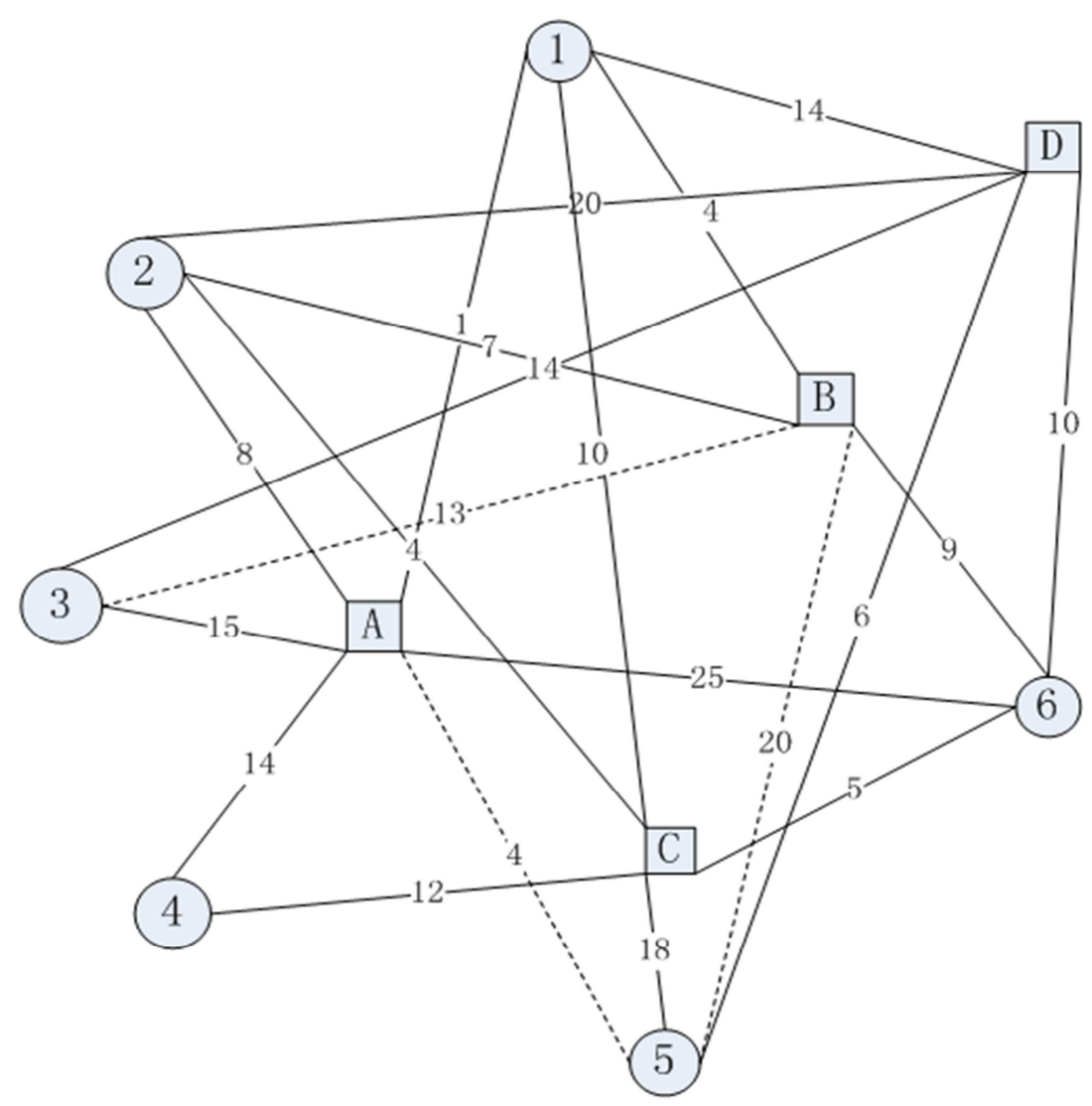

Fig. 3. The weighted network about the use of EVS between residential areas and workplaces.

First, the mathematical model is established and solved by LINGO software, and the exact optimal solution is $y_{1}=4, y_{2}=1, y_{3}=3, y_{4}=4, x_{1}=x_{2}=\ldots=x_{6}=0$, i.e., the charging stations are established in node A, B, C, D and the number of charging piles are respectively $4,1,3,4$. The maximum number of EVs covered by charging stations is 200 .
We also use the heuristic algorithm solving the model as follows:

Calculate $R_{i}, R_{j}, c u_{i}, u_{j}, v_{i}, c v_{j}$ and the results are listed in Table 2.

Table 2. Locating the first charging station.

\begin{tabular}{llllllllllll}
\hline $\boldsymbol{g}_{\boldsymbol{i}}$ or $\boldsymbol{h}_{\boldsymbol{j}}$ & $\mathbf{6 0}$ & $\mathbf{7 0}$ & $\mathbf{8 0}$ & $\mathbf{1 0 0}$ & $\mathbf{6 0}$ & $\mathbf{9 0}$ & $\mathbf{8 0}$ & $\mathbf{1 0 0}$ & $\mathbf{7 0}$ & $\mathbf{9 0}$ \\
\hline Nodes & 1 & 2 & 3 & 4 & 5 & 6 & $\mathrm{~A}$ & $\mathrm{~B}$ & $\mathrm{C}$ & $\mathrm{D}$ \\
$R_{i}$ or $R_{j}$ & 29 & 39 & 42 & 26 & 48 & 49 & 67 & 53 & 49 & 64 \\
$u_{i}$ or $v_{j}$ & 3 & 4 & 5 & 3 & 5 & 5 & 4 & 3 & 3 & 4 \\
$c u_{i}$ or $c v_{j}$ & 6.21 & 7.18 & 9.52 & 11.54 & 6.25 & 9.18 & 4.78 & 5.66 & 3.96 & 5.62 \\
\hline
\end{tabular}

From Table 2, the first charging station is built in node $\mathrm{C}$ and the number of charging piles established in node $\mathrm{C}$ is 3 , the costs of establishing 3 charging piles are $3 \times 70=210<1000$, i.e., $y_{3}=3$.

Remove all edges covered by node $\mathrm{C}$ and recalculate $R_{i}, R_{j}, u_{i}, v_{j}, c u_{i}, c v_{j}$. The results are shown in Table 3.

Table 3. Locating the second charging station.

\begin{tabular}{llllllllll}
\hline $\boldsymbol{g}_{\boldsymbol{i}}$ or $\boldsymbol{h}_{\boldsymbol{j}}$ & $\mathbf{6 0}$ & $\mathbf{7 0}$ & $\mathbf{8 0}$ & $\mathbf{1 0 0}$ & $\mathbf{6 0}$ & $\mathbf{9 0}$ & $\mathbf{8 0}$ & $\mathbf{1 0 0}$ & $\mathbf{9 0}$ \\
\hline Nodes & 1 & 2 & 3 & 4 & 5 & 6 & $\mathrm{~A}$ & $\mathrm{~B}$ & $\mathrm{D}$ \\
$R_{i}$ or $R_{j}$ & 19 & 35 & 42 & 14 & 30 & 45 & 67 & 53 & 64 \\
$u_{i}$ or $v_{j}$ & 2 & 4 & 5 & 2 & 3 & 5 & 4 & 3 & 4 \\
$c u_{i}$ or $c v_{j}$ & 6.32 & 8 & 9.52 & 14.29 & 6 & 10 & 4.78 & 5.66 & 5.62 \\
\hline
\end{tabular}


From Table 3, the second charging station is built in node A, and the number of charging piles established in node A is 4 , the costs of establishing 4 charging piles are $4 \times 80=320$, total costs used in nodes $\mathrm{C}$ and A are $210+320=530<1000$, i.e., $y_{1}=4$.

Remove all edges covered by node $\mathrm{A}$ and recalculate $R_{i}, R_{j}, u_{i}, v_{j}, c u_{i}, c v_{j}$. The results are shown in Table 4.

Table 4. Locating the third charging station.

\begin{tabular}{lllllllll}
\hline $\boldsymbol{g}_{\boldsymbol{i}}$ or $\boldsymbol{h}_{\boldsymbol{j}}$ & $\mathbf{6 0}$ & $\mathbf{7 0}$ & $\mathbf{8 0}$ & $\mathbf{1 0 0}$ & $\mathbf{6 0}$ & $\mathbf{9 0}$ & $\mathbf{1 0 0}$ & $\mathbf{9 0}$ \\
\hline Nodes & 1 & 2 & 3 & 4 & 5 & 6 & $\mathrm{~B}$ & $\mathrm{D}$ \\
$R_{i}$ or $R_{j}$ & 18 & 27 & 27 & 0 & 26 & 20 & 53 & 64 \\
$u_{i}$ or $v_{j}$ & 2 & 3 & 3 & 3 & 2 & 3 & 4 \\
$c u_{i}$ or $c v_{j}$ & 6.67 & 7.78 & 8.89 & & 6.92 & 9 & 5.66 & 5.62 \\
\hline
\end{tabular}

From Table 4, the third charging station is built in node $\mathrm{D}$ and the number of charging piles are 4 , the costs of establishing 4 charging piles are $4 \times 90=360$, the total costs used in node C, A, D are $530+360=890<1000$, i.e., $y_{4}=4$. Because $R_{4}=0$, so node 4 is not considered in the following steps.

Remove all edges covered by node A and recalculate $R_{i}, R_{j}, u_{i}, v_{j}, c u_{i}, c v_{j}$. The results are shown in Table 5.

Table 5. Locating the forth charging station.

\begin{tabular}{|c|c|c|c|c|c|c|c|}
\hline$g_{i}$ or $h_{j}$ & 60 & 70 & 80 & 100 & 60 & 90 & 100 \\
\hline Nodes & 1 & 2 & 3 & 4 & 5 & 6 & B \\
\hline$R_{i}$ or $R_{j}$ & 14 & 20 & 14 & 0 & 6 & 11 & 53 \\
\hline$u_{i}$ or $v_{j}$ & 2 & 2 & 2 & & 1 & 2 & 1 \\
\hline$c u_{i}$ or $c v_{j}$ & 8.57 & 7 & 11.43 & & 10 & 16.36 & 5.66 \\
\hline
\end{tabular}

From Table 5, the forth charging station is built in node B and the number of charging piles are 4 , the costs of establishing 4 charging piles are $4 \times 100=400$. Since the total costs used in node A, B, C, D >1000, so only one charging pile can be established in node $\mathrm{B}$ under the budget (1000), i.e., $y_{2}=1$.

Since the remaining budget 10 dollar is not enough for establishing another charging piles in any candidate charging station, so we end the algorithm.

By now, we have found four charging stations and the number of charging piles in each station. The four charging stations are all in workplaces (A, B, C, D), and the charging stations can satisfy 200 EVs' charging requirements. The result obtained by heuristic algorithm is the same as that obtained by Lingo software, which is a global optimal solution.

Because the number of residents who are willing to buy EVs as commuter tools is 233, and the four charging stations can cover $200 \mathrm{EVs}$, so the coverage rate is $\frac{200}{233} \approx 85.84 \%$.

\section{Discussion and Conclusions}

The paper studies the EV charging station location problem under the condition that the EVs are regarded as commuter tools. The weighted network is constructed according to the number of EVs used for commuting and commuting distance. Charging station location problem was transformed into maximum covering location problem of weighted network, and the integer nonlinear programming model is established. A heuristic algorithm is designed to solve the mathematical model. In the end, we do simulation on an example. The result shows that the mathematical model and algorithm is an effective way to solve the EV charging station location problem.

During the study of EV charging station location problem, only one phase of EV charging station location problem is considered, long-term planning about government's multi-stage investment strategy is not considered in the paper. Actually, the government will allocate a certain funds every year for establishing charging stations. So the long-term investment strategy for multi-stage charging stations establishing is necessary. In the process of multi-stage investment of charging stations construction, how to determine the locations of charging stations in each phase is the problem we will study in the future.

\section{Acknowledgements}

This work was supported by the National Natural Science Foundation of China (11131009, 71540028, F012408), the Funding Project of Beijing high level innovation and entrepreneurship program teaching teacher, Beijing Key Laboratory (NO: BZ0211), Beijing Intelligent Logistics System Collaborative Innovation Center and Major Research Project of Beijing Wuzi University. 


\section{References}

[1] X. Tang, J. Liu, Y. Liu, H. Feng, L. Xie, W. Ma, "Electric vehicles charging station planning based on computational geometry method," Automation of Electric Power Systems. Vol. 36, No. 8, 2012, pp. 24-30.

[2] H. Jia, S. Xie, X. Shao, S. Huang, "The location model of the electric vehicle charging station based on simulated annealing algorithm," Shanxi Electronic Technology. Vol. 3, 2013, pp. $27-33$

[3] T. Chen, Z. Wei, S. Wu, G. Sun, Y. Wei, X. Xu, "Distribution network planning by considering siting and sizing of electric vehicle charging stations," Proceeding of the CSU-EPSA. Vol. 25, No. 3, 2013, pp. 2-5.

[4] Z. Huang, J. Yang, "Problem of locating electric vehicle refueling stations with service capacity," Industrial Engineering and Management. Vol. 20, No. 5, 2015, pp. 111-118.

[5] X. Sun, K. Liu, Z. Zuo, "A spatiotemporal location model for locating electric vehicle charging stations," Progress in Geography. Vol. 31, No.6, 2012, pp. 687-692.

[6] S. Ge, L. Feng, H. Liu, L. Wang, "Planning of electric vehicle charging stations considering users' convenience," Advanced
Technology of Electrical Engineering and Energy. Vol. 33, No. 2, 2014, pp. 71-75.

[7] Y. Chu, L. Ma, H. Zhang, "Location-allocation and its algorithms for gradual covering vehicle charging stations," Mathematics in Practice and Theory. Vol. 45, No. 10, 2015, pp. 102-106.

[8] G. Chen, Z. Mao, J. Li, D. Wang, H. Zhou, K. Sun, "Multi-objective optimal planning of electric vehicle charging stations considering carbon emission," Automation of Electric Power Systems. Vol. 38, No. 17, 2014, pp. 49-53.

[9] J. Cavadas, G. Correia, J. Gouveia, “A MIP model for locating slow-charging stations for electric vehicles in urban areas according for driver tours," Transportation Research. 2015, pp. 188-201.

[10] F. He, Y. Yin, J. Zhou, "Deploying public charging stations for electric vehicles on urban road networks," Transportation Research. 2015, pp. 227-240.

[11] M. Hosseini. S. Hassani, "Selecting optimal location for electric recharging stations with queue," Journal of civil Engineering. Vol. 19, No. 7, 2015, pp. 2271-2280.

[12] A. Johannes, "Optimizing charging station locations for urban taxi providers," Transportation Research. Vol. 85, 2016, pp. 233-246. 